\title{
Blood purification treatment initiated at the time of sepsis diagnosis effectively attenuates serum HMGB1 upregulation and improves patient prognosis
}

\author{
SHIXIANG ZHENG ${ }^{1}$, QINYONG WENG ${ }^{1}$, WENWEI WU ${ }^{1}$ and GUOHUA DING ${ }^{2}$ \\ ${ }^{1}$ Division of Critical Care Medicine, Union Hospital of Fujian Medical University, Fuzhou, Fujian 350001; \\ ${ }^{2}$ Division of Nephrology, Renmin Hospital of Wuhan University, Wuhan, Hubei 430000, P.R. China
}

Received August 1, 2016; Accepted May 5, 2017

DOI: $10.3892 /$ etm.2017.4854

\begin{abstract}
The aim of the present study was to investigate the increase in serum and urine levels of high mobility group box protein 1 (HMGB1) during sepsis and the effect of blood purification treatments on HMGB1 levels and patient prognosis. A total of 40 intensive care patients with sepsis were randomly assigned to different groups ( $n=10$ per group): $\mathrm{A}$ control group (sepsis group), a continuous renal replacement treatment (CRRT) group, a hemoperfusion (HP) group and an HP+CRRT group. The blood purification treatments using HP and/or CRRT were performed immediately after the diagnosis of sepsis. HMGB1 levels were measured using ELISA, and Acute Physiology and Chronic Health Evaluation (APACHE) II scores and 30-day survival rates were evaluated. Relative to a healthy control group $(n=10)$, HMGB1 levels were observed to be significantly upregulated during sepsis $(\mathrm{P}<0.05)$. Following the initiation of sepsis, serum HMGB1 continued to increase in the sepsis group and was significantly elevated at $24 \mathrm{~h}(\mathrm{P}<0.05)$, whereas urine HMGB1 levels decreased significantly at 12 and $24 \mathrm{~h}(\mathrm{P}<0.05)$. Serum HMGB1 levels were significantly positively correlated with APACHE II scores $(\mathrm{r}=0.7284, \mathrm{P}<0.01)$ and significantly negatively correlated with urine HMGB1 levels $(\mathrm{r}=-0.5103, \mathrm{P}=0.026)$. Serum HMGB1 levels were significantly reduced in the HP and HP+CRRT groups by 12 and $24 \mathrm{~h}$ following the initiation of treatment (both $\mathrm{P}<0.05$ ). Changes in the urine HMGB1 level differed in each group. Relative to the sepsis group, the APACHE II scores of all blood purification groups were significantly reduced $(\mathrm{P}<0.05)$ and the 30-day survival rate of the HP+CRRT group was significantly increased $(\mathrm{P}=0.0107)$. The results of the present study indicate that blood purification initiated at the
\end{abstract}

Correspondence to: Dr Shixiang Zheng, Division of Critical Care Medicine, Union Hospital of Fujian Medical University, 29 Xinquan Road, Fuzhou, Fujian 350001, P.R. China

E-mail: jeromesuper@126.com

Key words: sepsis, high mobility group box protein 1, blood purification, excretion, prognosis point of diagnosis in patients with sepsis may attenuate serum HMGB1 upregulation, promote urinary excretion of HMGB1 and improve the prognosis of sepsis.

\section{Introduction}

Sepsis is a systematic inflammatory response to infection and is the leading cause of death in intensive care units (ICUs) $(1,2)$. During sepsis, many types of danger-associated molecular patterns (DAMPs) that mediate inflammatory responses and organ failure are released into the blood (3-5). High mobility group box-1 (HMGB1) is a conserved non-histone DNA-binding protein that serves as a type of DAMP during many pathological processes, including sepsis and disseminated intravascular coagulation (6). It has previously been reported that high levels of HMGB1 are released into the blood as sepsis occurs, which subsequently mediate the inflammatory response. In a previous study by our group, it was demonstrated that HMGB1 enters the urine and mediates inflammation by transforming renal epithelial cells into inflammatory promoters during sepsis (7). However, the extent to which HMGB1 is released into the blood and enters the urine remains unclear.

As techniques improve, blood purification methods, including continuous renal replacement therapy (CRRT) and hemoperfusion (HP), are becoming more commonly used as treatment methods for sepsis $(8,9)$. Previous reports have suggested that the attenuation of several pro-inflammatory cytokines, such as interleukins and tumor necrosis factors, may be the primary underlying mechanism of blood purification as an effective treatment for sepsis $(10,11)$. However, other studies have reported no association between the attenuation of cytokines and the use of blood purification techniques during sepsis $(12,13)$. The exact mechanism of blood purification as a cure for sepsis remains unclear.

Previous studies have suggested that increased levels of serum HMGB1 may be associated with a poorer outcome in sepsis patients (SPs), and that clinical treatments that reduce HMGB1 may be effective for the treatment of sepsis $(14,15)$. HMGB1 is an important DAMP that amplifies the systemic inflammatory response, and blood purification is an effective cure for sepsis. Therefore, it is important to determine the 
extent to which serum and urine HMGB1 levels are elevated during sepsis, and the influence of blood purification treatments initiated immediately after sepsis diagnosis on serum and urine HMGB1 levels and sepsis prognosis. The present study aimed to answer these questions.

\section{Materials and methods}

Study population. A total of 40 adult SPs with different sepsis severities, who were admitted to the ICU of Union Hospital (Fujian Medical University, Fuzhou, China) between January and December 2015, were enrolled in the present study. Informed consent was obtained from all patients or their legal representatives, and all study protocols were approved by the Ethics Committee of Fujian Medical University and followed regulations in accordance with the Declaration of Helsinki. Sepsis was defined according to the criteria of the American College of Chest Physicians/Society of Critical Care Medicine (16) and diagnosed as sepsis, severe sepsis or septic shock. The baseline characteristic data of the enrolled patients are presented in Table I. A total of 10 healthy volunteers (5 males and 5 females) who were absent of any disease were enrolled as a healthy control group (mean age, $57.25 \pm 13.78$ years).

Exclusion criteria. The exclusion criteria were as follows: Patients $<18$ years old, patients with life-threatening water electrolyte/acid-base balance disorder within the first $24 \mathrm{~h}$ of the ICU stay, patients with end stage renal disease receiving any type of blood purification therapy, pregnancy, receipt of immunosuppressive therapy or human immunodeficiency virus infection, current participation in any other clinical trial and inability to be evaluated effectively during the study period.

Clinical treatment. Immediately after a diagnosis of sepsis was confirmed, patients were randomly assigned to a control (no intervention) group without any apheresis treatment (sepsis group, $\mathrm{n}=10)$, a CRRT group ( $\mathrm{n}=10)$, an HP group $(\mathrm{n}=10)$ and an HP+CRRT group $(n=10)$. Blood purification treatments were initiated immediately after informed consent was obtained. A 12 Fr double lumen catheter was placed in the right femoral vein of each patient to establish vascular access. All blood purification treatments were conducted using a PrismaFlex system (Baxter International, Inc., Deerfield, IL, USA). Blood purification data are presented in Table II. With the exception of the blood purification treatments performed immediately following the diagnosis of sepsis, all other treatments were consistent among the groups. All patients were treated according to the Guidelines for Management of Severe Sepsis and Septic Shock (Surviving Sepsis Campaign International 2013) (17). The primary end point of the study was either patient survival at 30 days after enrollment or patient mortality during the 30-day period.

ELISA tests. Serum and urine HMGB1 levels were measured using a Human HMGB1 ELISA kit (E-EL-H1554c, Elabscience Biotechnology Co., Ltd., Wuhan, China) according to the manufacturer's protocol. Briefly, blood samples were obtained from peripheral vascular, blood and urine specimens $(\sim 2 \mathrm{ml})$ were collected at the initiation of treatment and at 12 and $24 \mathrm{~h}$ thereafter. Blood specimens were maintained at room temperature for $2 \mathrm{~h}$, and serum samples were collected prior to centrifugation. Serum and urine samples were centrifuged at $4^{\circ} \mathrm{C}$ in a swinging bucket rotor at $1,000 \mathrm{x}$ g for $20 \mathrm{~min}$, and the supernatant was collected and measured using the ELISA kit.

Data collection. Following patient enrollment, the demographic data, sepsis type (sepsis, severe sepsis or septic shock), infection sites and biological data of patients were recorded. Acute Physiology and Chronic Health Evaluation (APACHE) II (18) scores were assigned at the initiation of treatment and $24 \mathrm{~h}$ later. All patients were observed for 30 days, and the mortality rate during this period was recorded. Information regarding patient medical history and preexisting conditions was obtained by examining the patients' previous medical records.

Statistical analyses. Statistical analyses were performed using GraphPad Prism version 6 (GraphPad Software Inc., La Jolla, CA, USA). Data are presented as the mean \pm standard deviation. All variables were tested for normal distribution. Differences between groups were evaluated by analysis of variance and Tukey's multiple comparison tests were performed. Correlations between the serum and urine HMGB1 levels and APACHE II scores of the SP groups at the initiation of treatment $(0 \mathrm{~h})$ were calculated using Pearson's correlation analysis. Time-to-event data were compared using the Gehan-Breslow-Wilcoxon test for survival analysis. $\mathrm{P}<0.05$ was considered to indicate a statistically significant difference.

\section{Results}

Serum and urine HMGBI levels in different groups. The serum and urine HMGB1 levels of patients in different groups were measured using ELISA. The results indicated that upon diagnosis of sepsis, both the serum and urine HMGB1 levels were significantly increased in all SP groups when compared with the healthy control group $(\mathrm{P}<0.05$; Fig. 1).

Serum and urine HMGB1 levels in the sepsis group at different time points. To investigate the changes in serum and urine HMGB1 levels within the first $24 \mathrm{~h}$ of sepsis, serum and urine HMGB1 levels of patients in the sepsis group were measured at the time of sepsis diagnosis $(0 \mathrm{~h})$ and at 12 and $24 \mathrm{~h}$ thereafter. Serum HMGB1 levels increased gradually within the first $24 \mathrm{~h}$ of sepsis, and the serum HMGB1 level at $24 \mathrm{~h}$ after diagnosis was significantly increased compared with that at $0 \mathrm{~h}(\mathrm{P}<0.05$; Fig. 2A). By contrast, the urine HMGB1 level was significantly decreased at 12 and $24 \mathrm{~h}$ compared with that at $0 \mathrm{~h}(\mathrm{P}<0.05$; Fig. 2B).

Correlations between the serum HMGB1 level, urine HMGBI level and APACHE II score. Associations between serum and urine HMGB1 levels and APACHE II scores were analyzed using Pearson's correlation analysis. The results indicated a significant negative correlation between the serum HMGB1 level and urine HMGB1 level in the SP groups ( $\mathrm{r}=-0.5103$, $\mathrm{P}=0.026$; Fig. 3A). By contrast, the serum HMGB1 level and APACHE II score were significantly positively correlated in patients with sepsis ( $r=0.7284, \mathrm{P}<0.01$; Fig. 3B). 
Table I. Baseline characteristics of patients prior to the initiation of treatment.

\begin{tabular}{|c|c|c|c|c|c|}
\hline \multirow[b]{2}{*}{ Variables } & \multicolumn{4}{|c|}{ Group } & \multirow[b]{2}{*}{ P-value } \\
\hline & Sepsis & CRRT & $\mathrm{HP}$ & $\mathrm{HP}+\mathrm{CRRT}$ & \\
\hline \multicolumn{6}{|l|}{ Demographic data } \\
\hline Age, years & $56.60 \pm 14.16$ & $61.50 \pm 13.16$ & $59.10 \pm 20.34$ & $57.70 \pm 15.62$ & 0.91 \\
\hline Male, $\%$ & $4(40)$ & $6(60)$ & $6(60)$ & $5(50)$ & $\mathrm{N} / \mathrm{A}$ \\
\hline MAP, $\mathrm{mmHg}$ & $51.85 \pm 9.84$ & $56.45 \pm 16.27$ & $54.06 \pm 14.00$ & $55.77 \pm 12.57$ & 0.87 \\
\hline \multicolumn{6}{|l|}{ Type of infection, n (\%) } \\
\hline Pulmonary & $4(40)$ & $6(60)$ & $4(40)$ & $5(50)$ & N/A \\
\hline Abdominal & $1(10)$ & $3(30)$ & $2(20)$ & $1(10)$ & N/A \\
\hline Skin and soft tissue & $2(20)$ & $0(0)$ & $2(20)$ & $3(30)$ & N/A \\
\hline Other & $3(30)$ & $1(1)$ & $2(20)$ & $1(10)$ & N/A \\
\hline \multicolumn{6}{|l|}{ Biological data } \\
\hline $\mathrm{WBC}, \mathrm{x} 10^{3} / \mathrm{mm}^{3}$ & $17.07 \pm 5.06$ & $22.32 \pm 9.23$ & $24.03 \pm 8.56$ & $19.59 \pm 6.23$ & 0.14 \\
\hline $\mathrm{HB}, \mathrm{g} / 1$ & $83.53 \pm 5.09$ & $90.32 \pm 10.74$ & $83.72 \pm 6.40$ & $84.09 \pm 10.06$ & 0.22 \\
\hline PCT, ng/ml & $20.52 \pm 5.10$ & $16.98 \pm 7.83$ & $23.20 \pm 7.76$ & $18.05 \pm 8.03$ & 0.24 \\
\hline ALB, $g / 1$ & $28.93 \pm 6.49$ & $28.05 \pm 7.32$ & $27.28 \pm 7.77$ & $26.72 \pm 5.31$ & 0.90 \\
\hline \multicolumn{6}{|l|}{ Type of sepsis, n (\%) } \\
\hline Sepsis & $2(20)$ & $2(20)$ & $3(30)$ & $1(10)$ & $\mathrm{N} / \mathrm{A}$ \\
\hline Severe sepsis & $7(70)$ & $6(60)$ & $6(60)$ & $5(50)$ & N/A \\
\hline Septic shock & $1(10)$ & $2(20)$ & $1(10)$ & $4(40)$ & N/A \\
\hline \multicolumn{6}{|l|}{ Pathogens, n (\%) } \\
\hline MRSA & $3(30)$ & $4(40)$ & $3(30)$ & $4(40)$ & N/A \\
\hline Pseudomonas aeruginosa & $2(20)$ & $4(40)$ & $3(30)$ & $3(30)$ & N/A \\
\hline Acinetobacter baumanii & $5(50)$ & $3(30)$ & $4(40)$ & $4(40)$ & N/A \\
\hline Other bacteria & $2(20)$ & $1(10)$ & $0(0)$ & $2(20)$ & N/A \\
\hline \multicolumn{6}{|l|}{ Organ Dysfunction, n (\%) } \\
\hline None & $2(20)$ & $2(20)$ & $3(30)$ & $1(10)$ & N/A \\
\hline Lung & $8(80)$ & $7(70)$ & $7(70)$ & $9(90)$ & N/A \\
\hline Kidney & $1(10)$ & $2(20)$ & $1(10)$ & $3(30)$ & N/A \\
\hline Gastrointestinal & $0(0)$ & $1(10)$ & $0(0)$ & $1(10)$ & N/A \\
\hline Liver & $0(0)$ & $0(0)$ & $1(10)$ & $1(10)$ & N/A \\
\hline
\end{tabular}

Demographic and biological data are presented as the mean \pm standard deviation. CRRT, continuous renal replacement treatment; HP, hemoperfusion; N/A, not applicable; MAP, mean arterial pressure; WBC, white blood cells; HB, hemoglobin; PCT, procalcitonin; ALB, human serum albumin; MRSA, Methicillin-resistant Staphylococcus aureus.

Changes in the serum and urine HMGB1 levels in the blood purification groups. To investigate the effects of various treatments on serum and urine HMGB1 levels, serum and urine samples were collected from the different SP groups and measured using ELISA kits. The results indicated that serum HMGB1 was significantly increased in the sepsis group at $24 \mathrm{~h}$ compared with that of 0 and $12 \mathrm{~h}$. Serum and urine HMGB1 levels were significantly reduced in the HP and HP+CRRT groups at 12 and $24 \mathrm{~h}$ following the initiation of treatment $(\mathrm{P}<0.05$; Fig. 4). In the CRRT group, a significant reduction in serum HMGB1 was observed $24 \mathrm{~h}$ after the initiation of treatment $(\mathrm{P}<0.05)$, while urine HMGB1 levels did not differ significantly between 0 and $24 \mathrm{~h}$. The serum and urine HMGB1 levels of different groups were compared with those of the sepsis group at various time points. The results indicated that the serum and urine HMGB1 levels of the HP and HP+CRRT groups were significantly lower compared with that of the sepsis group at 12 and $24 \mathrm{~h}$ following the initiation of treatment $(\mathrm{P}<0.05$; Fig. 4). By contrast, only the serum HMGB1 level was significantly decreased in the CRRT group compared with the sepsis group at $24 \mathrm{~h}(\mathrm{P}<0.05)$. In addition, urine HMGB1 levels in the CRRT and HP+CRRT groups $24 \mathrm{~h}$ after the initiation of treatment were significantly higher compared with those at $12 \mathrm{~h}(\mathrm{P}<0.05$; Fig. 4).

Changes in APACHE II scores in the different groups. To determine the effects of different blood purification treatments on the short-term outcome of patients, the APACHE II scores of patients were measured. The APACHE II scores of the HP+CRRT group were significantly higher compared with all 
Table II. Blood purification therapy data for the different groups.

\begin{tabular}{lllcccc}
\hline Group & \multicolumn{1}{c}{ Filter set } & \multicolumn{1}{c}{ Time, $\mathrm{h}$} & BF, ml/min & CTD, ml/kg/h & Pre/post & Heparin, IU/kg/h \\
\hline Sepsis & N/A & N/A & N/A & N/A & N/A & N/A \\
CRRT & M100 Set & 24 & 180 & 35 & $2: 1$ & 5 \\
HP & Adsorba 300 & 2.5 & 180 & N/A & N/A & 5 \\
HP+ & M100 Set & HP+CRRT & 180 & 35 & $2: 1$ & 5 \\
CRRT & +Adsorba 300 & 2.5+CRRT 21.5 & & & & \\
\hline
\end{tabular}

CRRT, continuous renal replacement treatment; HP, hemoperfusion; N/A, not applicable; BF, blood flow; CTD, CRRT treatment dose; Pre, pre-substitution; Post, post-substitution.

A

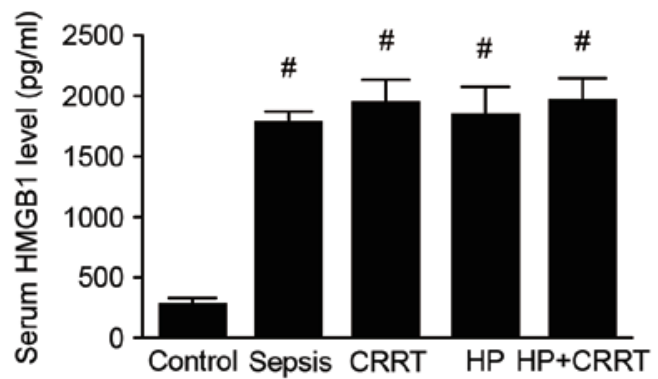

B

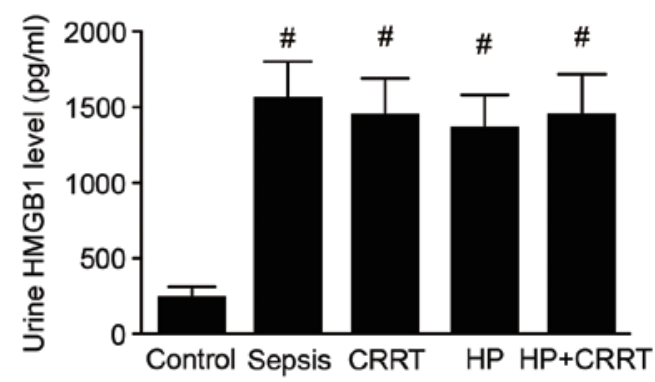

Figure 1. Serum and urine HMGB1 levels upon diagnosis of sepsis. (A) Serum and (B) urine levels of HMGB1 were measured by ELISA in the different patient groups prior to the initiation of treatment. Data are presented as the mean \pm standard deviation. ${ }^{*} \mathrm{P}<0.05$ vs. Control. HMGB1, high mobility group box protein 1; CRRT, continuous renal replacement treatment; HP, hemoperfusion.
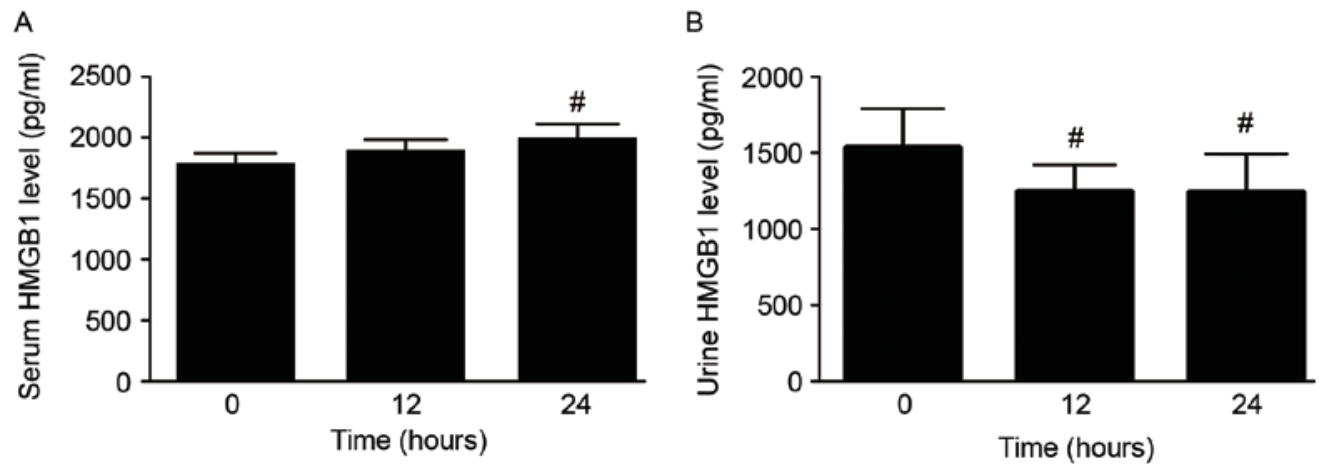

Figure 2. Changes in serum and urine HMGB1 levels over $24 \mathrm{~h}$. (A) Serum and (B) urine levels of HMGB1 in the sepsis (no apheresis intervention) group at different time points. Data are presented as the mean \pm standard deviation. ${ }^{*} \mathrm{P}<0.05$ vs. 0 -h value. HMGB1, high mobility group box protein 1 .
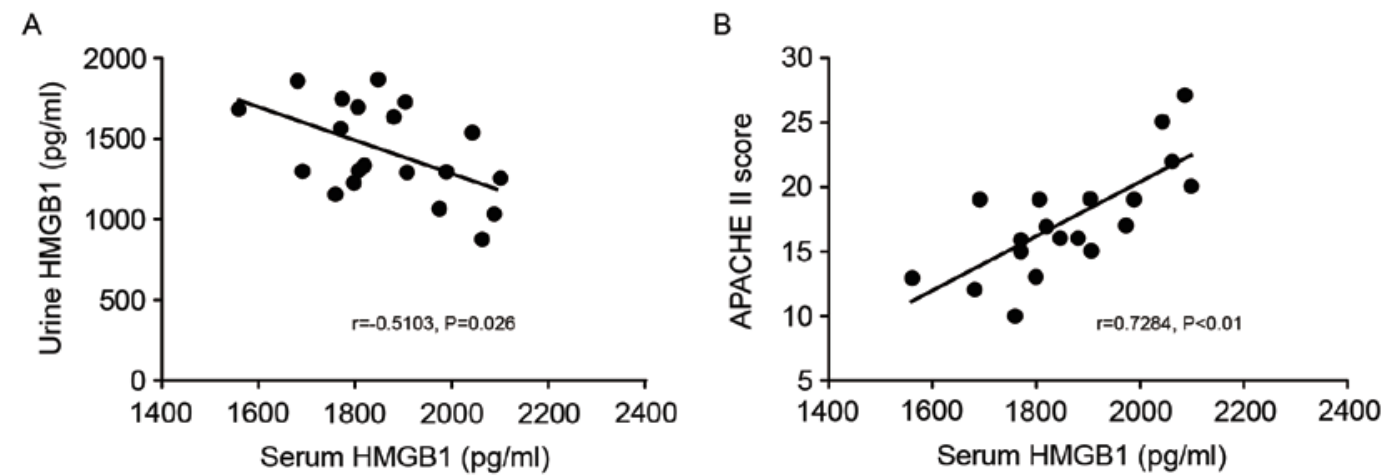

Figure 3. Association of serum HMGB1 level with urine HMGB1 level and APACHE II score. Correlations were identified between (A) urine and serum HMGB1 levels, and (B) APACHE II score and serum HMGB1 level in the sepsis (no apheresis intervention) group. HMGB1, high mobility group box protein 1; APACHE II, Acute Physiology and Chronic Health Evaluation II. 
A

Sepsis $\square \mathrm{CRRT} \backsim \mathrm{HP} \quad \mathrm{HP}+\mathrm{CRRT}$

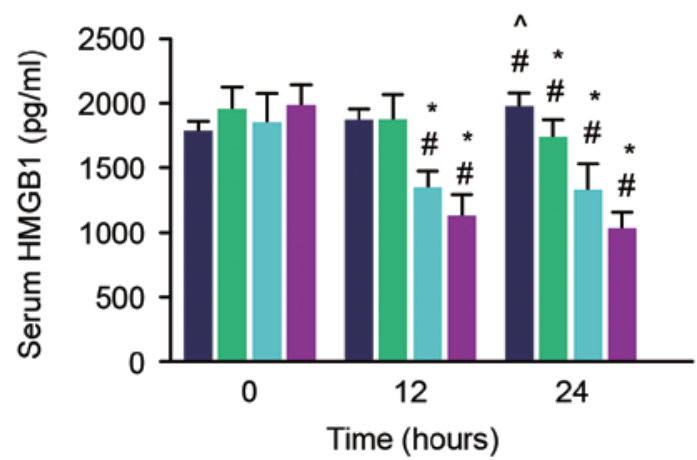

B

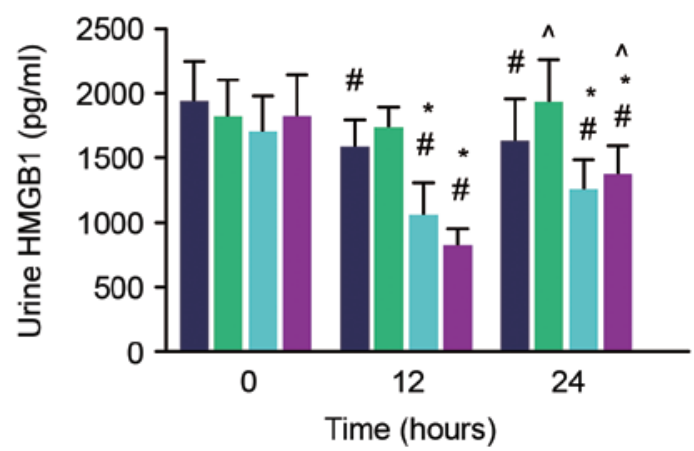

Figure 4. Changes in serum and urine HMGB1 levels in the different patient groups over $24 \mathrm{~h}$. (A) Serum and (B) urine levels of HMGB1 were measured by ELISA at different time points after the initiation of treatments. Data are presented as the mean \pm standard deviation. ${ }^{~} \mathrm{P}<0.05$ vs. 0 -h value; ${ }^{*} \mathrm{P}<0.05$ vs. sepsis group; ${ }^{\wedge} \mathrm{P}<0.05$ vs. 12 -h value. HMGB1, high mobility group box protein 1 ; CRRT, continuous renal replacement treatment; HP, hemoperfusion.

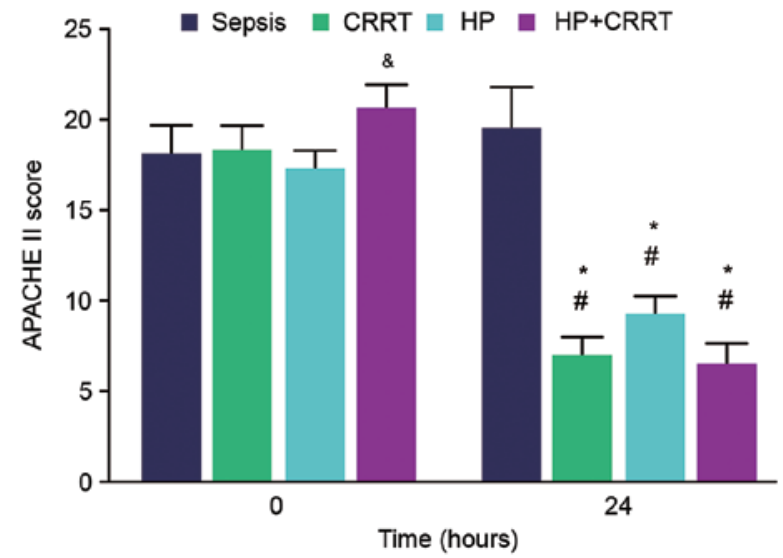

Figure 5. APACHE II scores of patients in different groups upon initiation of treatment $(0 \mathrm{~h})$ and $24 \mathrm{~h}$ thereafter. Data are presented as the mean \pm standard deviation. ${ }^{\&} \mathrm{P}<0.05$ vs. all other groups at $0 \mathrm{~h}$; ${ }^{*} \mathrm{P}<0.05$ vs. 0 -h value; ${ }^{*} \mathrm{P}<0.05$ vs. sepsis group at $24 \mathrm{~h}$. APACHE II, Acute Physiology and Chronic Health Evaluation II; CRRT, continuous renal replacement treatment; HP, hemoperfusion.

other SP groups at the initiation of treatment $(\mathrm{P}<0.05$; Fig. 5). At $24 \mathrm{~h}$ post-initiation of treatment, the APACHE II scores of patients in the CRRT, HP and HP+CRRT groups were significantly decreased compared with those at $0 \mathrm{~h}(\mathrm{P}<0.05$; Fig. 5). Furthermore, at $24 \mathrm{~h}$ post-initiation of treatment, the APACHE II scores of the CRRT, HP and HP+CRRT groups were significantly lower compared with the sepsis group $(\mathrm{P}<0.05$; Fig. 5).

Survival outcome. After a 30-day follow-up period, the survival rates of the different SP groups were as follows: Sepsis group, $20 \%$; CRRT group, $60 \%$; HP group, $60 \%$; and HP+CRRT group, $80 \%$ (Fig. 6). Despite the higher survival rates of the CRRT and HP groups compared with that of the sepsis group, survival analysis demonstrated that the improvements were not statistically significant $(\mathrm{P}=0.0519$ and $\mathrm{P}=0.0832$, respectively). However, the results indicated that the 30 -day survival rate of the HP+CRRT group was significantly higher compared with the sepsis group $(\mathrm{P}=0.0107)$, suggesting that combined treatment with HP+CRRT significantly improves the survival outcome of patients.

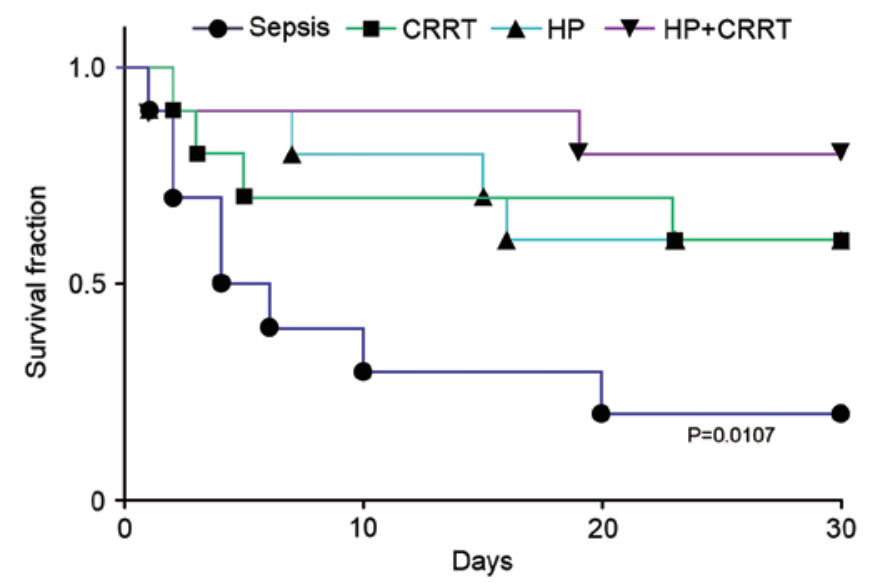

Figure 6. Survival curves demonstrating the 30-day survival rates of patients in different groups. CRRT, continuous renal replacement treatment; HP, hemoperfusion.

\section{Discussion}

Sepsis is a major clinical syndrome associated with a high mortality rate in hospitalized patients (19). It has previously been demonstrated that extracellular HMGB1 serves as an important DAMP and activates the innate immune system (20-22). Animal studies have indicated that HMGB1 may be both actively and passively released into the blood during sepsis, and may act as an important proinflammatory cytokine that mediates the inflammatory response $(6,7)$. Pyroptosis and exocytosis of secretory lysosomes by activated immune cells are the primary processes that allow intercellular HMGB1 to reach extracellular spaces $(23,24)$. In the present study, it was observed that serum HMGB1 was significantly increased in SPs, and continued to rise during the development of sepsis. This is consistent with previous research (25) and suggests that serum HMGB1 serves an important role in the development of sepsis. A previous study also indicated that in specific pathological processes, including systemic lupus erythematosus (26), serum HMGB1 was able to infiltrate the urine; therefore, urine HMGB1 levels may be closely related to the severity of such diseases. The results of the present study 
indicated that urine HMGB1 levels were also significantly increased in SPs. HMGB1 may be excreted in the urine $(26,27)$, which suggests that urine is an important route for HMGB1 excretion during sepsis. Furthermore, results of the present study indicated that urine HMGB1 levels were significantly decreased at 12 and $24 \mathrm{~h}$ following confirmed sepsis diagnosis, suggesting that extracellular excretion of HMGB1 via the urine decreases as sepsis progresses.

To further investigate the association between serum HMGB1 levels and the severity of sepsis, a correlation analysis of serum HMGB1 levels and APACHE II scores was performed. The results suggested that patients with more severe sepsis had higher levels of serum HMGB1. Furthermore, results of the correlation analysis indicated that the urine HMGB1 level was negatively correlated with the serum HMGB1 level. These results suggest that as the clearance of HMGB1 decreases, the accumulation of serum HMGB1 is correlated with the aggravation of sepsis.

It is important to identify an effective method that attenuates serum HMGB1 levels and enhances the clearance of HMGB1 during the early stages of sepsis. Previous studies have indicated that blood purification techniques are able to directly and indirectly reduce the levels of proinflammatory cytokines in the blood (28-30). Similar to cytokines, the molecular weight of HMGB1 is relatively low $(\sim 30 \mathrm{kDa})(31)$, and thus serum HMGB1 may also be effectively cleared by specific blood purification techniques initiated upon diagnosis of sepsis. In the present study, three different blood purification techniques were used (CRRT, HP and HP+CRRT) and the serum and urine HMGB1 levels were measured at different time points. Despite enhanced clearance of serum HMGB1 by the blood purification techniques, the results indicated that HP and HP+CRRT were more effective than CRRT. As activated carbon can absorb hydrophobic proteins, the HP technique may directly clear serum HMGB1; further research is required to confirm this. However, as the cut-off molecular weight of the hemofilter used in the current study was $\sim 15-20 \mathrm{kDa}$, as reported previously (32), it was concluded that HMGB1 may not be directly cleared by CRRT. It was therefore hypothesized that CRRT may enhance the clearance of serum HMGB1 by increasing the excretion of HMGB1 via the urine. The urine and serum HMGB1 levels were measured at different time points and the results suggested that, although all urine and serum HMGB1 levels were markedly attenuated $12 \mathrm{~h}$ after the initiation of treatment, urine HMGB1 levels in the CRRT and HP+CRRT groups were significantly increased at 24 compared with $12 \mathrm{~h}$. CRRT is able to continuously stabilize the internal environment and eliminate low molecular weight metabolic waste products of patients, including creatinine and urea nitrogen $(33,34)$, which suggests that CRRT treatment enhances the excretion of HMGB1 via the urine.

The effects of different blood purification treatments on the prognosis of SPs were investigated. The results indicated that although early HP+CRRT was the most effective method for reducing the APACHE II scores of patients, all three treatments effectively attenuated the APACHE II scores. These findings paralleled the reduction in serum HMGB1 levels in each group. As APACHE II score has been correlated with the short-term prognosis of SPs (35-37), the present data indicated that all of the investigated treatments were able to improve the short-term prognosis of SPs. Furthermore, results of the 30-day survival analysis indicated that, although all blood purification treatments were able to increase the survival rate of SPs at early-stage sepsis, only the HP+CRRT treatment significantly improved patients' 30 -day survival rate.

The present study was a randomized single-center clinical trial with a limited sample size; as such, multi-center studies are now warranted to verify the present findings. Future studies are also required to determine the exact mechanism underlying HMGB1 excretion, and the mechanisms by which blood purification techniques affect serum/urine HMGB1 levels and the prognosis of sepsis.

In conclusion, the present study demonstrated that serum and urine HMGB1 levels were significantly increased during sepsis, and the greatest increases in serum HMGB1 levels were positively correlated with the severity of sepsis and reduced excretion of HMGB1 in the urine. Blood purification treatments, particularly HP+CRRT, initiated immediately upon diagnosis of sepsis, effectively attenuated serum HMGB1 levels, promoted the excretion of HMGB1 via the urine and improved the prognosis of sepsis. Sepsis is a complicated clinical syndrome that lacks specific treatment methods, and the removal of common proinflammatory cytokines does not fully explain the effects of blood purification on sepsis (38). The results of the present study may provide a novel perspective regarding the mechanism of blood purification in sepsis, and may aid to develop a novel treatment strategy for sepsis.

\section{References}

1. Vincent JL and Atalan HK: Epidemiology of severe sepsis in the intensive care unit. Br J Hosp Med (Lond) 69: 442-443, 2008.

2. Zhou J, Qian C, Zhao M, Yu X, Kang Y, Ma X, Ai Y, Xu Y, Liu D, An Y, et al: Epidemiology and outcome of severe sepsis and septic shock in intensive care units in mainland China. PLoS One 9: e107181, 2014.

3. Kakihana Y, Ito T, Nakahara M, Yamaguchi K and Yasuda T: Sepsis-induced myocardial dysfunction: Pathophysiology and management. J Intensive Care 4: 22, 2016.

4. Timmermans K, Kox M, Scheffer GJ and Pickkers P: Danger in the intensive care unit: Damps in critically ill patients. Shock 45: 108-116, 2016.

5. Kang JW, Kim SJ, Cho HI and Lee SM: DAMPs activating innate immune responses in sepsis. Ageing Res Rev 24: 54-65, 2015.

6. Liaw PC, Ito T, Iba T, Thachil J and Zeerleder S: DAMP and DIC: The role of extracellular DNA and DNA-binding proteins in the pathogenesis of DIC. Blood Rev 30: 257-261, 2016.

7. Zheng S, Pan Y, Wang C, Liu Y, Shi M and Ding G: HMGB1 turns renal tubular epithelial cells into inflammatory promoters by interacting with TLR4 during sepsis. J Interferon Cytokine Res 36: 9-19, 2016.

8. Sun J, Shi S, Wang Q, Yu K and Wang R: Continuous hemodiafiltration therapy reduces damage of multi-organs by ameliorating of HMGB1/TLR4/NFkB in a dog sepsis model. Int J Clin Exp Pathol 8: 1555-1564, 2015.

9. Mitaka C, Masuda T, Kido K, Uchida T, Abe S, Miyasho T, Tomita $\mathrm{M}$ and Inada E: Polymyxin B hemoperfusion prevents acute kidney injury in sepsis model. J Surg Res 201: 59-68, 2016.

10. Yimin H, Wenkui Y, Jialiang S, Qiyi C, Juanhong S, Zhiliang L, Changsheng H, Ning L and Jieshou L: Effects of continuous renal replacement therapy on renal inflammatory cytokines during extracorporeal membrane oxygenation in a porcine model. J Cardiothorac Surg 8: 113, 2013.

11. Pohl J, Papathanasiou M, Heisler M, Stock P, Kelm M, Hendgen-Cotta UB, Rassaf T and Luedike P: Renal replacement therapy neutralizes elevated MIF levels in septic shock. J Intensive Care 4: 39, 2016.

12. Lukaszewicz AC and Payen D: Purification methods: A way to treat severe acute inflammation related to sepsis? Crit Care 17: $159,2013$. 
13. Park JT, Lee H, Kee YK, Park S, Oh HJ, Han SH, Joo KW, Lim CS, Kim YS, Kang SW, et al: High-dose versus conventional-dose continuous venovenous hemodiafiltration and patient and kidney survival and cytokine removal in sepsis-associated acute kidney injury: A randomized controlled trial. Am J Kidney Dis 68: 599-608, 2016.

14. Gibot S, Massin F, Cravoisy A, Barraud D, Nace L, Levy B and Bollaert PE: High-mobility group box 1 protein plasma concentrations during septic shock. Intensive Care Med 33: 1347-1353, 2007.

15. Sundén-Cullberg J, Norrby-Teglund A, Rouhiainen A, Rauvala H, Herman G, Tracey KJ, Lee ML, Andersson J, Tokics L and Treutiger CJ: Persistent elevation of high mobility group box-1 protein (HMGB1) in patients with severe sepsis and septic shock. Crit Care Med 33: 564-573, 2005.

16. Bone RC, Balk RA, Cerra FB, Dellinger RP, Fein AM, Knaus WA, Schein RM and Sibbald WJ: Definitions for sepsis and organ failure and guidelines for the use of innovative therapies in sepsis. The ACCP/SCCM consensus conference committee. American college of chest physicians/society of critical care medicine. Chest 101: 1644-1655, 1992.

17. Schorr CA and Dellinger RP: The surviving sepsis campaign: Past, present and future. Trends Mol Med 20: 192-194, 2014.

18. Afessa B, Gajic O and Keegan MT: Severity of illness and organ failure assessment in adult intensive care units. Crit Care Clin 23 639-658, 2007.

19. Perner A, Rhodes A, Venkatesh B, Angus DC, Martin-Loeches I, Preiser JC, Vincent JL, Marshall J, Reinhart K, Joannidis M and Opal SM: Sepsis: Frontiers in supportive care, organisation and research. Intensive Care Med 43: 496-508, 2017.

20. Wu H, Steenstra R, de Boer EC, Zhao CY, Ma J, van der Stelt JM and Chadban SJ: Preconditioning with recombinant high-mobility group box 1 protein protects the kidney against ischemia-reperfusion injury in mice. Kidney Int 85: 824-832, 2014.

21. Chen YC, Statt S, Wu R, Chang HT, Liao JW, Wang CN, Shyu WC and Lee CC: High mobility group box 1-induced epithelial mesenchymal transition in human airway epithelial cells. Sci Rep 6: 18815, 2016.

22. LiG,Wu X, Yang L,He Y,Liu Y,Jin X and Yuan H:TLR4-mediated $\mathrm{NF}-\kappa \mathrm{B}$ signaling pathway mediates HMGB1-induced pancreatic injury in mice with severe acute pancreatitis. Int J Mol Med 37 99-107, 2016

23. Lamkanfi M, Sarkar A, Vande Walle L, Vitari AC, Amer AO, Wewers MD, Tracey KJ, Kanneganti TD and Dixit VM: Inflammasome-dependent release of the alarmin HMGB1 in endotoxemia. J Immunol 185: 4385-4392, 2010.

24. Lu B, Nakamura T, Inouye K, Li J, Tang Y, Lundbäck P, Valdes-Ferrer SI, Olofsson PS, Kalb T, Roth J, et al: Novel role of PKR in inflammasome activation and HMGB1 release. Nature 488: 670-674, 2012
25. Huang W, Tang Y and Li L: HMGB1, a potent proinflammatory cytokine in sepsis. Cytokine 51: 119-126, 2010.

26. Abdulahad DA, Westra J, Bijzet J, Dolff S, van Dijk MC Limburg PC, Kallenberg CG and Bijl M: Urine levels of HMGB1 in systemic lupus erythematosus patients with and without renal manifestations. Arthritis Res Ther 14: R184, 2012.

27. Leelahavanichkul A, Huang Y, Hu X, Zhou H, Tsuji T, Chen R, Kopp JB, Schnermann J, Yuen PS and Star RA: Chronic kidney disease worsens sepsis and sepsis-induced acute kidney injury by releasing high mobility group box protein-1. Kidney Int 80 : 1198-1211, 2011

28. Liu L and Ding G: Effects of different blood purification methods on serum cytokine levels and prognosis in patients with acute severe organophosphorus pesticide poisoning. Ther Apher Dial 19: 185-190, 2015.

29. Hongliang T, Rong Z, Xiaojing W, Rao S, Lun L, Jinhui T, Nong C and Kehu Y: The effects of continuous blood purification for SIRS/MODS patients: A systematic review and Meta-analysis of randomized controlled trials. ISRN Hematol 2012: 986795 , 2012.

30. Tsuchida K, Yoshimura R, Nakatani T and Takemoto Y: Blood purification for critical illness: Cytokines adsorption therapy. Ther Apher Dial 10: 25-31, 2006.

31. Shen $X$ and Li WQ: High-mobility group box 1 protein and its role in severe acute pancreatitis. World J Gastroenterol 21 1424-1435, 2015.

32. Menth M, Röckel A, Abdelhamid S, Fiegel P and Walb D: Low-molecular-weight protein-permeable cuprammonium rayon haemofilter. Nephrol Dial Transplant 7: 844-847, 1992.

33. Yessayan L, Yee J, Frinak S and Szamosfalvi B: Continuous renal replacement therapy for the management of acid-base and electrolyte imbalances in acute kidney injury. Adv Chronic Kidney Dis 23: 203-210, 2016.

34. Amlani GS: Continuous renal replacement therapy. J Pak Med Assoc 62: 276-280, 2012.

35. Gursel G and Demirtas S: Value of APACHE II, SOFA and CPIS scores in predicting prognosis in patients with ventilator-associated pneumonia. Respiration 73: 503-508, 2006.

36. Costa JI, Gomes do Amaral JL, Munechika M, Juliano Y and Bezerra Filho JG: Severity and prognosis in intensive care: Prospective application of the APACHE II index. Sao Paulo Med J 117: 205-214, 1999.

37. Knaus WA: APACHE 1978-2001: The development of a quality assurance system based on prognosis: Milestones and personal reflections. Arch Surg 137: 37-41, 2002.

38. Peng ZY, Wang HZ, Carter MJ, Dileo MV, Bishop JV, Zhou FH, Wen XY, Rimmelé T, Singbartl K, Federspiel WJ, et al: Acute removal of common sepsis mediators does not explain the effects of extracorporeal blood purification in experimental sepsis. Kidney Int 81: 363-369, 2012 